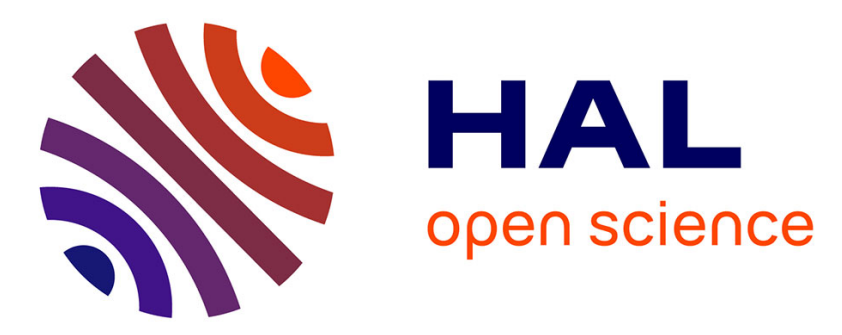

\title{
Caractérisation du régime des hautes eaux en débit-durée-fréquence. Application au contexte algérien
}

Eric Sauquet, G. Galea, M. Bessenasse

\section{To cite this version:}

Eric Sauquet, G. Galea, M. Bessenasse. Caractérisation du régime des hautes eaux en débit-duréefréquence. Application au contexte algérien. La Houille Blanche - Revue internationale de l'eau, 2004, 5, p. 80 - p. 85 . 10.1051/lhb:200405010 . hal-00450837

\section{HAL Id: hal-00450837 https://hal.science/hal-00450837}

Submitted on 27 Jan 2010

HAL is a multi-disciplinary open access archive for the deposit and dissemination of scientific research documents, whether they are published or not. The documents may come from teaching and research institutions in France or abroad, or from public or private research centers.
L'archive ouverte pluridisciplinaire HAL, est destinée au dépôt et à la diffusion de documents scientifiques de niveau recherche, publiés ou non, émanant des établissements d'enseignement et de recherche français ou étrangers, des laboratoires publics ou privés. 


\title{
Caractérisation du régime des hautes eaux en débit-durée-fréquence Application au contexte algérien ${ }^{1}$
}

\author{
Describing the flow regime by the Flood-Duration-Frequency approach \\ An application to the Saf-Saf river basin, Algeria
}

Eric Sauquet, Gilles Galéa

Cemagref Lyon, Unité de Recherche Hydrologie-Hydraulique,

3bis, quai Chauveau CP220, 69336 Lyon cedex 09, France

\section{Mohamed Bessenasse}

Université SAAD Dahleb (Blida), B.P 26 D, Hadjout, W. Tipaza, 42200 Algérie

This paper presents a Flood Frequency Analysis (FFA), which takes into account the notion of duration: the floodduration-frequency $(Q d F)$ method. $Q d F$ analysis is similar to the intensity-duration-frequency analysis commonly used for rainfall. The first part describes the analytical formulations. An application to the Saf-Saf River is performed. QdF curves are fitted. Synthetic Mono-Frequency Hydrographs (SMFHs) are then derived. The main advantage of these hydrographs is that all the characteristics of the SMFHs are related to the same return period. These design flood hydrographs are inputs for hydraulics modelling and simulation of sedimentation in Zardezas reservoir (Algeria).

\section{INTRODUCTION}

La caractérisation du régime hydrologique d'une rivière pour les besoins cognitifs ou opérationnels requiert la mise en œuvre de méthodes qui synthétisent une information riche mais parfois inextricable contenue dans les chroniques de débit. Pour les pluies, une des représentations la plus communément utilisée est donnée par les courbes IdF, Intensitédurée-Fréquence. Ce type d'approche est généralisable à d'autres variables, en hydrologie mais également dans d'autres domaines. Ainsi, Sobey et Orloff [1] adaptent le concept d'analyse en Intensité-durée-Fréquence à la houle.

La démarche $\operatorname{QdF}(Q:$ débit, $d$ : durée, $F$ : fréquence) s'inscrit dans une démarche de quantification probabiliste de l'aléa hydrologique de façon analogue à l'approche IdF. On s'intéresse non plus uniquement aux statistiques des valeurs maximales instantanées mais on étend l'analyse aux caractéristiques relatives à différentes durées pour affecter à un volume ou une intensité moyenne une fréquence ou période de retour. Cette représentation a été initiée par l'Institut

1. Ce texte a été présenté lors du colloque : «Gestion du risque Eau en pays semi-aride », SHF, ADENIT, ENIT, INAT, Tunis, 20-22 mai 2003.
d'Hydrologie de Wallingford [2] pour les crues et a connu depuis des développements multiples au Cemagref : l'approche par bassin de référence en crue [3] et en étiage [4] et récemment le modèle convergent $[5,6,7]$. Les courbes $\mathrm{QdF}$ peuvent être exploitées pour construire des hydrogrammes de projet qui présentent des caractéristiques en débit homogènes en fréquence.

L'objectif de cette communication est de présenter les derniers développements de l'analyse QdF et d'illustrer la méthode par une application au contexte algérien: l'oued Saf-Saf

\section{II — L'APPROCHE DÉBIT-DURÉE-FRÉQUENCE}

L'analyse QdF fournit une description fréquentielle théorique multi-durée des quantiles de crue ou d'étiage. Elle s'intéresse à deux variables hydrologiques représentatives du régime des hautes eaux et extraites de la chronique brute de débit :

- le débit-seuil dépassé en continu pendant la durée $d$, maximal sur l'épisode de crue, noté $Q d$ (fig. 1.a); 

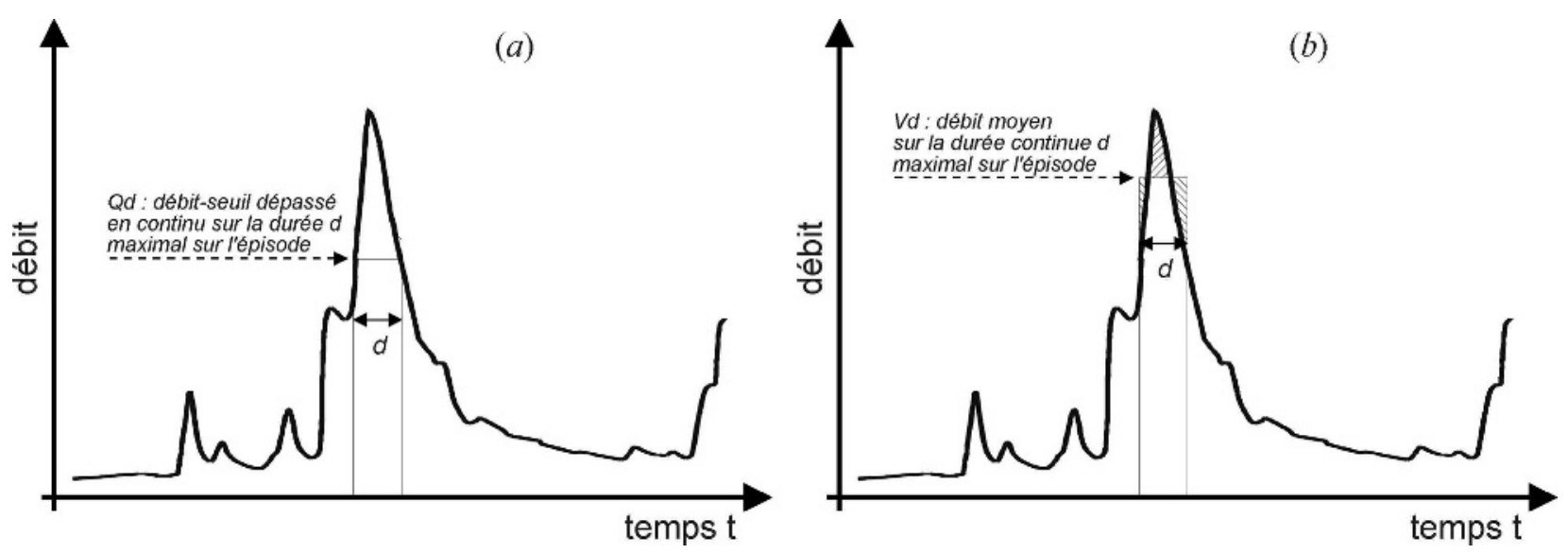

Figure 1 : Définition des variables hydrologiques.

- le débit moyen, moyenne glissante calculée sur la durée continue $d$, maximal sur l'épisode de crue, noté $V d$ (fig. 1.b).

Lorsque $d=0$, les deux variables sont égales au débit instantané maximal $Q I X$.

Les résultats se présentent généralement sous la forme d'un faisceau de courbes paramétrées par la durée $d$ dans le plan $(Q, T)$ ou transformé $(Q, \ln (T))$. Les courbes $\mathrm{QdF}$ décrivent les variations de $V d$ et $Q d$ en fonction de la durée $d$ et de la période de retour $T$.

Lorsque l'on dispose d'une série suffisamment longue, l'analyse se décline en deux phases :

- échantillonnage multidurée de valeurs supérieures à un seuil : on prendra soin de sélectionner des événements indépendants et de se rapprocher d'un processus d'occurrence stationnaire de type Poisson (principes de la méthode du renouvellement) ;

- ajustements d'une loi de probabilité permettant de définir la fréquence théorique de chaque épisode, exprimée en terme de période de retour $T$ sur les échantillons.

\section{II.1 Crues observables $(0,5 \leq T$ (an) $\leq 10)$}

L'ajustement d'un modèle probabiliste peut être effectué : - individuellement pour chaque durée $d$; on privilégie la distribution exponentielle dans le cas d'un échantillonnage par valeur supérieure à un seuil :

$$
V d(T)=a q_{d} \ln (T)+x_{0, d}
$$

avec :

- $a q_{d}$ : gradex des débits ou encore paramètre d'échelle de la loi ;

$-x_{0, d}:$ paramètre de position de la loi ;

ou :

- simultanément sur l'ensemble des durées par ajustement d'une fonction mathématique $V(d, T)$ et/ou $Q(d, T)$ sur les échantillons.

Une première manière d'aboutir à $V(d, T)$ et/ou $Q(d, T)$ consiste alors à utiliser les expressions mathématiques d'un modèle bibliothèque adimensionnel dit «de référence » [3]. Il s'agit de choisir le meilleur modèle parmi trois disponibles selon des critères hydro-météorologiques et de dénormer les formulations à l'aide de deux caractéristiques une en débit et l'autre en durée, toutes deux obtenues au site d'intérêt.
La seconde possibilité est d'appliquer le modèle QdF «convergent» [5]. Cette formulation repose sur une propriété observée sur un grand nombre de chroniques hydrologiques traitées : les ajustements $V d(T)$ tendent à se croiser en un même point vers les très faibles périodes de retour et sur une propriété d'affinité orthogonale des lois de probabilité théoriques des quantiles relatifs à différentes durées [6]. Le modèle QdF convergent permet de synthétiser dans une formulation analytique unique les quantiles de crue relatifs à différentes durées, indépendamment des lois de probabilité retenues :

$$
V(d, T)=\frac{V(0, T)}{1+d / \Delta}
$$

avec :

- $V(0, T)$ : distribution théorique des débits de pointe $(Q I X)$ consolidée par modélisation ;

$-\Delta$ : paramètre à caler, homogène à un temps et relié à la dynamique de crue.

On parle d'ajustement consolidé $V(0, T)$ car toutes les durées participent à l'estimation des paramètres de la distribution théorique. Les distributions de durée $d$ (2) se déduisent de la distribution modélisée des débits de pointe $V(0, T)$, connaissant $\Delta$.

Plusieurs approches peuvent être envisagées pour estimer $\Delta$ et obtenir simultanément une distribution consolidée $V(0, T)[5,8,9,10]$. La procédure privilégiée fait intervenir un processus de calage de $\Delta$ par itérations successives sur le principe des moindres carrés. Elle présente l'avantage d'être simple d'application et économe en hypothèses.

De l'expression (2), et compte tenu de la propriété suivante :

$$
V(d, T)=\frac{1}{d} \int_{0}^{d} Q(\delta, T) d \delta
$$

on déduit une formulation pour l'estimation des quantiles de débits seuils $Q(d, T)$ :

$$
Q(d, T)=\frac{V(0, T)}{(1+d / \Delta)^{2}}
$$


Dans le cas d'un ajustement $V(0, T)$ à la loi exponentielle, il vient :

$$
V(d, T)=\frac{x_{0}+a_{0} \ln (T)}{1+d / \Delta}
$$

et

$$
Q(d, T)=\frac{x_{0}+a_{0} \ln (T)}{(1+d / \Delta)^{2}}
$$

- où les paramètres $a_{0}, x_{0}$ et $\Delta$ sont à déterminer.

\section{II.2 Extrapolation aux grandes périodes de retour} $(10<T($ an $) \leq 1000)$

Pour prédéterminer les quantiles de fréquence rare, on introduit une information pluviométrique qui prend progressivement le relais pour l'estimation des quantiles à partir de $T=10$ ans, seuil fréquentiel d'extrapolation. Le modèle d'extrapolation des distributions choisi est une variante du modèle du gradex esthétique [11] :

$V(d, T)=V(d, 10)+C(d) \cdot \ln \left(1+\frac{A(d)}{C(d)} \cdot \frac{T-10}{10}\right) \cdot Q I X A 10$ (7) avec :

- $V(d, 10):$ donné par $(5)$;

- QIXA10 : débit de pointe décennal égal à $V(0,10)$;

- $A(d)$ : gradex implicite des débits relatifs à la durée $d$ fourni par (5), cf. ultérieurement ;

$-C(d)$ : gradex des pluies maximales implicite d'un modèle QdF de référence [3].

\section{APPLICATION}

\section{III.1 Présentation du bassin-versant}

L'analyse QdF présentée ici s'inscrit dans une étude de la sédimentation dans la retenue de Zardezas de la région de Skikda (Algérie) [12]. Elle a pour objectif de fournir les données d'entrée sous la forme d'hydrogrammes de projet à une modélisation numérique pour prédire la formation et l'évolution des dépôts de sédiments à l'amont du barrage de Zardezas.

A l'amont immédiat de la retenue, une station hydrométrique a mesuré les débits moyens journaliers sur la période entre 1968 et 1993 et fournit certains hydrogrammes de crues parmi les plus fortes de manière plus précise. Le bassin versant contrôlé par la station a une surface de $345 \mathrm{~km}^{2}$ (fig. 2).

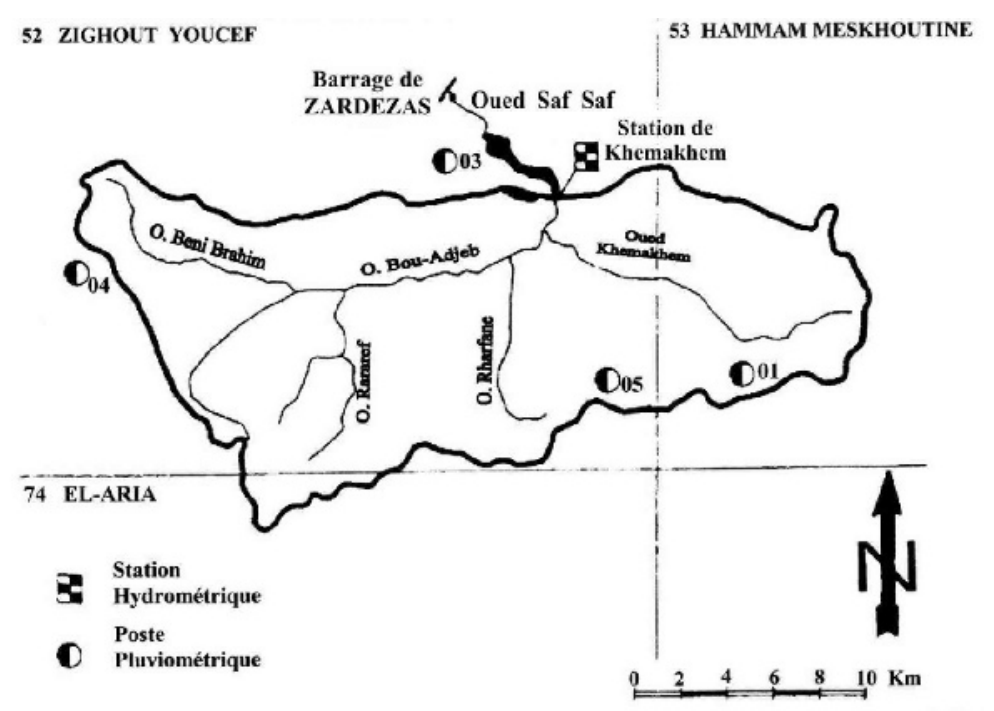

Figure 2 : Bassin versant alimentant l'oued Saf-Saf.

La méthode du GRADEX [13] sera la méthode de référence pour l'estimation des quantiles de grande période de retour. Elle requiert une information pluviométrique. Les seules données de pluie disponibles sont fournies par un poste situé à l'exutoire du bassin versant ( $\mathrm{n}^{\circ} 3$ en fig. 2). Le poste pluviométrique situé à l'exutoire du bassin versant n'est pas du meilleur choix mais le seul possible consenti par le service d'exploitation de la retenue et dont le gradex annuel des pluies maximales de $24 \mathrm{~h}\left(G p_{24 \mathrm{~h}}=24,7 \mathrm{~mm}\right)$ a été porté à notre connaissance.

\section{III.2 Distributions modélisées et quantiles de crue selon l'approche convergente}

Les trois paramètres, $a_{0}, x_{0}$ et $\Delta$ sont ajustés sur l'ensemble des quantiles expérimentaux relatifs aux durées $d$ étudiées. Pour le bassin du Saf-Saf, nous obtenons les expressions suivantes :

$$
V(d, T)=\frac{110 \ln T+109}{1+d / 15.8}
$$


et, s'en déduit :

$$
Q(d, T)=\frac{110 \ln T+109}{(1+d / 15,8)^{2}}
$$

Pour l'extrapolation aux quantiles rares, le gradex implicite des débits s'obtient à partir de (8) :

$$
A(d)=\frac{110}{Q X X A 10 \cdot(1+d / 15,8)}
$$

et le gradex implicite des pluies est celui du modèle de référence le plus adapté aux bassins rapides :

$$
C^{\prime}(d)=\frac{1}{0,569 \frac{d}{D}+0,69}+0,046
$$

avec $D$ : durée de SOCOSE [14].
La durée caractéristique de crue $D$ du bassin versant du Saf-Saf estimée à partir des quelques crues observées disponibles, est voisine de $4 \mathrm{~h}(D=4 \mathrm{~h})$. Diverses études de régionalisation des crues, sous différents climats, ont montré une forte liaison entre $\Delta$ et $D$. Le rapport $\Delta / D$ est relativement constant (de l'ordre de 3,5) [15]. Pour le bassin du SafSaf, le ratio est de 3,95 , ce qui confirme la valeur estimée $D$.

La figure 3 représente les échantillons observés et les ajustements obtenus par modélisation QdF pour les crues, de faible (5) ou de grande (7) période de retour. Sont reportés également les échantillons des crues extrapolées déterminées à partir $\mathrm{du}$ gradex des pluies maximales connu $\left(G p_{24 \mathrm{~h}}=24,7 \mathrm{~mm}\right)$.

Deux événements exceptionnels en pointe de période de retour théorique proche de la centennale ont été mesurés. Leurs volumes sur $24 \mathrm{~h}$ excèdent $V(24 \mathrm{~h}, 100)$. Ils ont une fréquence beaucoup plus rare que la centennale, voire très rare pour l'un d'eux. Pour le pas de temps de $24 \mathrm{~h}$, l'extrapolation décrite par (7) vérifie les quantiles rares expérimentaux estimés à partir du gradex des pluies maximales observées de $24 \mathrm{~h}$ et est ainsi validée.

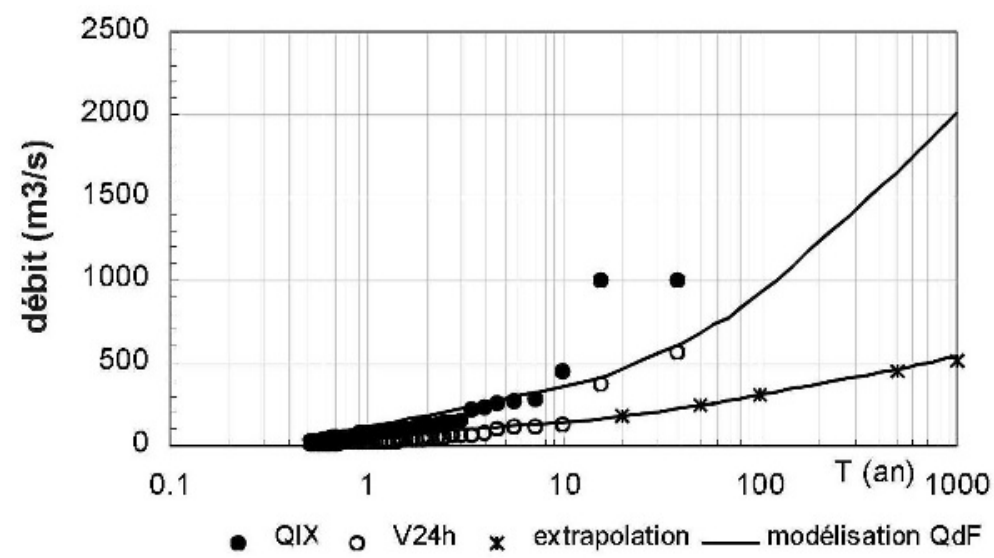

Figure 3 : Modélisation QdF du régime des crues.

La forme d'extrapolation esthétique au regard des deux valeurs extrêmes de débit quotidien mesurées est critiquable. Un choix objectif entre gradex esthétique et gradex brut [13] nécessiterait une étude régionale des régimes de crues et des pluies associées. Ce contexte d'étude n'est pas l'objet de notre propos et l'approche hydrologique du bassin du SafSaf présentée est essentiellement un premier exemple d'application en climat semi-aride du concept QdF convergent développé en climat tempéré. Le choix du gradex esthétique (7) pour le bassin du Saf-Saf nous a permis de donner une estimation des quantiles rares en tenant compte d'un indice de saturation du sol selon le ratio $A(d) / C(d)$. Pour la durée $d$ de $24 \mathrm{~h}$ ce ratio est de 0,44 , ce qui est relativement éloigné de 1 , valeur pour laquelle le modèle d'extrapolation (7) est équivalent à celui du GRADEX d'EDF-DTG [13]. A défaut de toute information régionale, la valeur de l'indice de saturation de 0.44 peut apporter une certaine justification au choix du gradex esthétique. Ceci peut être aussi rapproché du coefficient d'écoulement inter-annuel relativement faible. En effet, pour une hauteur de précipitation moyenne de $940 \mathrm{~mm} / \mathrm{an}$ l'apport moyen inter-annuel à la retenue est de 45 millions de $\mathrm{m}^{3}$ ( 3 ou 4 plus fortes crues annuelles), soit un coefficient d'écoulement inter-annuel de $14 \%$ [12]. Par ailleurs, l'information pluviométrique dont nous disposions ne nous permettait pas de caler une forme d'extrapolation généralisée avec la durée $d$ et nous avons donc utilisé $C(d)$ du modèle de référence [3] dont la typologie des crues intègre un gradex esthétique implicite qui vérifie les quantiles rares de $24 \mathrm{~h}$ (fig. 3).

Les valeurs des débits moyens $V(d, T)$ et débits seuils $Q(d, T)$, issues de l'ajustement aux modèles analytiques, sont respectivement reportées dans les tableaux 1 et 2 . 
Tableau 1. Quantiles de crues de débit moyen modélisés.

\begin{tabular}{|c|c|c|c|c|c|c|c|c|c|c|}
\hline \multicolumn{11}{|c|}{ Quantiles $V(d, T)\left(\mathrm{m}^{3} / \mathrm{s}\right)$} \\
\hline \multirow{2}{*}{$d(\mathbf{h})$} & \multicolumn{10}{|c|}{$T$ (an) } \\
\hline & 0,5 & 1 & 2 & 5 & 10 & 20 & 50 & 100 & 500 & 1000 \\
\hline 0 & 32,8 & 109 & 185 & 286 & 362 & 462 & 684 & 925 & 1658 & 2013 \\
\hline 4 & 26,2 & 87,0 & 148 & 228 & 289 & 366 & 523 & 679 & 1116 & 1320 \\
\hline 6 & 23,8 & 79,1 & 134 & 207 & 263 & 332 & 469 & 602 & 968 & 1137 \\
\hline 12 & 18,6 & 62,0 & 105 & 163 & 206 & 259 & 359 & 453 & 702 & 816 \\
\hline 24 & 13,0 & 43,3 & 73,7 & 114 & 144 & 181 & 247 & 307 & 465 & 537 \\
\hline 48 & 8,1 & 27,0 & 46,0 & 71,0 & 89,9 & 113 & 154 & 191 & 289 & 333 \\
\hline
\end{tabular}

Tableau 2. Quantiles de crues de débit seuil dépassé modélisés.

\begin{tabular}{|c|c|c|c|c|c|c|c|c|c|c|}
\hline \multicolumn{11}{|c|}{ Quantiles $Q(d, T)\left(\mathrm{m}^{3} / \mathrm{s}\right)$} \\
\hline \multirow{2}{*}{$d(\mathbf{h})$} & \multicolumn{10}{|c|}{$T$ (an) } \\
\hline & 0,5 & 1 & 2 & 5 & 10 & 20 & 50 & 100 & 500 & 1000 \\
\hline 0 & 32,8 & 109 & 185 & 286 & 362 & 462 & 684 & 925 & 1658 & 2013 \\
\hline 4 & 20,9 & 69,5 & 118 & 182 & 231 & 290 & 401 & 504 & 769 & 887 \\
\hline 6 & 17,2 & 57,3 & 97,4 & 151 & 191 & 239 & 324 & 400 & 589 & 672 \\
\hline 12 & 10,6 & 35,3 & 60,0 & 92,6 & 117 & 146 & 193 & 233 & 329 & 370 \\
\hline 24 & 5,20 & 17,2 & 29,3 & 45,2 & 57,3 & 71,2 & 94,6 & 114 & 163 & 185 \\
\hline 48 & 2,02 & 6,71 & 11,4 & 17,6 & 22,3 & 28,2 & 40,0 & 51,4 & 82,3 & 96,5 \\
\hline
\end{tabular}

\section{III.3 Définition des hydrogrammes de projet}

Gilard [16] construit un Hydrogramme Synthétique MonoFréquence HSMF à partir d'une estimation des quantiles de débit seuil (fig.4). Il suppose la montée de l'hydrogramme en croissance linéaire avec le temps. Le maximum est atteint à l'instant $D$, durée de SOCOSE. La pointe de crue est définie par le débit $Q I X A T$ instantané maximal de la période de retour $T$ choisie. Pour différentes durées $d$, on construit la courbe de récession de telle sorte que le débit $Q(T, d)$ en décrue se situe à une distance $d$ du point d'ordonnée $Q(T, d)$ de la phase de montée. L'avantage de l'HSMF est de garantir que toutes les caractéristiques de l'hydrogramme sont rattachées à une même fréquence d'occurrence.
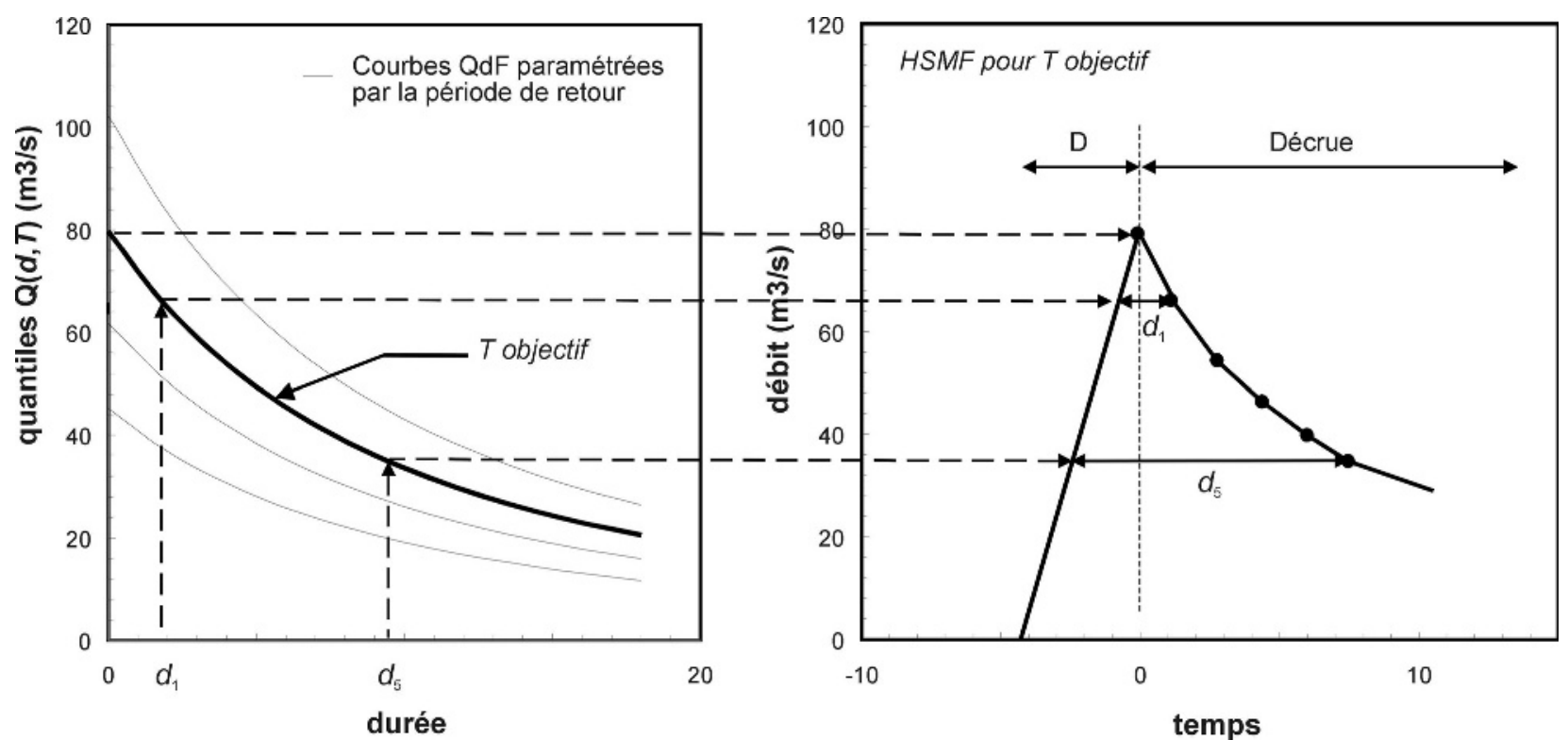

Figure 4 : Principe de construction d'un Hydrogramme Synthétique MonoFréquence associé à la période de retour $T$ objectif à partir des courbes $\mathrm{QdF}$. 
L'hydrogramme de projet pour le Saf-Saf est défini par un temps de montée $t m$ de $4 \mathrm{~h}(D=4 \mathrm{~h})$ et la courbe de récession s'appuie sur les quantiles $Q(d, T)$ relatifs aux durées $d$ du tableau 2. L'hydrogramme tracé en figure 5 a la propriété d'être homogène en période de retour 10 ans quelle que soit la variable $V d$ ou $Q d$ considérée.

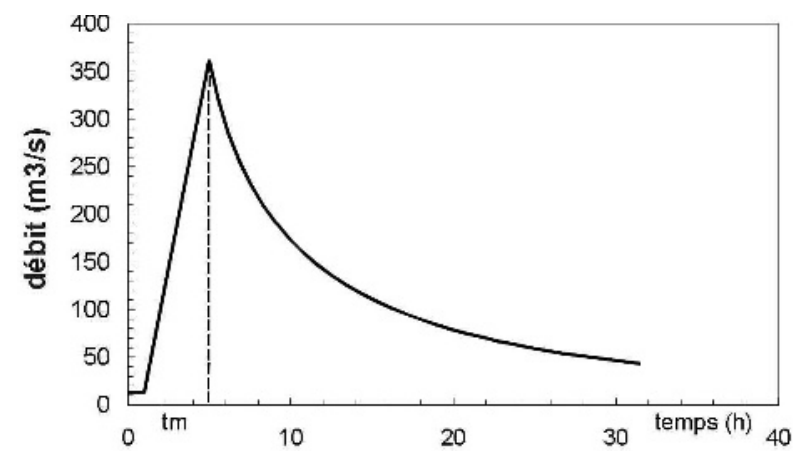

Figure 5 : Hydrogramme Synthétique Mono-Fréquence relatif à la période de retour 10 ans.

\section{V $\square$ CONCLUSION}

La présente communication s'intéresse à la description des régimes des crues à l'aide des courbes $\mathrm{QdF}$ en débit-duréefréquence. La formulation analytique de ces courbes est présentée. Simple d'application, elle requiert un choix non imposé de distribution théorique et la connaissance d'un paramètre $\Delta$ lié à la dynamique de crue et restitue les quantiles de débits moyens et débits seuils sur toute durée d'intérêt. Les courbes QdF ont permis de définir des hydrogrammes de projet dont les caractéristiques en débit sont relatives à la même période de retour. Cette approche a été appliquée au bassin versant du Saf-Saf en Algérie et une bonne cohérence avec la méthode du gradex a été observée pour prédire les quantiles de débits moyens de durée de $24 \mathrm{~h}$. Ceci est une première confirmation de l'applicabilité en climat semiaride de la méthode QdF convergent établie en climat dit tempéré. De cette analyse, ont été extraits des hydrogrammes de projet, les HSMFs, qui synthétisent le régime hydrologique des crues du bassin. Introduits dans une modélisation numérique, ils ont permis de suivre et reproduire l'évolution de la sédimentation dans la retenue de Zardezas.

\section{BIBLIOGRAPHIE}

[1] Sobey R.J. et OrLOFF L.S. (1999). - Intensity-duration-frequency summaries for wave climate. Coastal Engineering, 36 (1), 37-58.
[2] NerC (1975). - Estimation of flood volumes over different durations. Flood Study Report. Vol. 1, chap. 5, 243-264.

[3] Galéa G. et Prudhomme C. (1997). - Notions de base et concepts utiles pour la compréhension de la modélisation synthétique des régimes de crue des bassins versants au sens des modèles QdF. Revue des Sciences de l'Eau, 1, 83-101.

[4] Galéa G., Mercier G. et Adler M.J. (1999). - Modèles débit-durée-fréquence d'étiage, concept et usage pour une approche régionale des régimes de basses eaux des bassins hydrographiques de la Loire (France) et du Crisu-Alb (Roumanie). Revue des Sciences de l'Eau, 12 (1), 93-122.

[5] Javelle P., Ouarda T.B.M.J., Lang M., Bobée B. Galéa G. et GRÉSILLON J.-M. (2002). - Development of regional floodduration-frequency curves based on the index-flood method. Journal of Hydrology, 258, 249-259.

[6] Javelle P. (2001). - Caractérisation du régime des crues : le modèle débit-durée-fréquence convergent. Approche locale et régionale, thèse de doctorat de l'Institut National Polytechnique de Grenoble, $1^{\mathrm{er}}$ mars 2001, 268 p.

[7] Galéa G., Javelle P. et Chaput N. (2000). - Un modèle débit-durée-fréquence pour caractériser le régime d'étiage d'un bassin versant. Revue des Sciences de l'Eau, 13 (4), 421-440.

[8] Koutsoyiannis D., Kozonis D. et Manetas A. (1998). - A mathematical framework for studying rainfall intensity-duration-frequency relationships. Journal of Hydrology, 206 (1-2), $118-135$.

[9] MEUnieR M. (2001). - Regional flow-duration-frequency model for the tropical island of Martinique. Journal of Hydrology, 247 (1-2), 31-53.

[10]Javelle P., Ouarda T.B.M.J. et BobéE B. (2003). - Spring flood analysis using the Flood-duration-Frequency approach: Application to the Provinces of Quebec and Ontario, Canada. Hydrological Processes, Special Issue (accepté).

[11]Michel C. et Oberlin G. (1987). - Seuil d'application de la méthode du GRADEX. La Houille Blanche, n³ 3, 199-203.

[12]Bessenasse M., Kettab A., Paquier A., Galéa G. et Ramez P. (2003). - Simulation numérique de la sédimentation dans les retenues de barrages : cas de la retenue de Zardezas, Algérie. Revue des Sciences de l'Eau, 16 (1), 103 - 122.

[13]Guillot P. et Duband D. (1967). - La méthode du GRADEX pour le calcul de la probabilité des crues à partir des pluies. Journées de la SHF (septembre), question 1, rapport 7, Paris.

[14]CTGREF (1980). - Synthèse nationale sur les crues des petits bassins, fascicule 2 : la méthode SOCOSE. Informations Techniques $\mathrm{n}^{\circ} 38,2$ (juin 1980).

[15]Sauquet E., Javelle P. et Le Clerc S. (2003). - Description des régimes hydrologiques des hautes eaux : nouvelle formulation pour l'analyse en débit-durée-fréquence et applications en ingénierie. Ingénieries EAT, 34, 3-16.

[16]GILARD O. (1998). - Les bases techniques de la méthode Inondabilité. Cemagref Editions, Antony, 207 p. 